\title{
Effect of Osmolality on the Pharmacokinetic Interaction between Apple Juice and Atenolol in Rats
}

\author{
Yuta Funai, ${ }^{1}$ Yoshiyuki Shirasaka, ${ }^{1}$ Marika Ishihara, Miyuki Takemura, Kazuki Ichijo, \\ Hisanao Kishimoto, and Katsuhisa Inoue
}

School of Pharmacy, Tokyo University of Pharmacy and Life Sciences, Hachioji, Tokyo, Japan

Received September 13, 2018; accepted January 7, 2019

\section{ABSTRACT}

A recent clinical study reported that the ingestion of apple juice (AJ) markedly reduced the plasma concentration of atenolol; however, our in vitro study showed that atenolol may not be a substrate of organic anion transporting polypeptide 2B1 (OATP2B1), so this AJ-atenolol interaction cannot be explained by inhibition of OATP2B1. On the other hand, we more recently showed that the solution osmolality influences gastrointestinal (GI) water volume, and this may indirectly affect intestinal drug absorption. In this study, we examined whether the osmolality dependence of water dynamics can account for AJ-atenolol interactions by evaluating the GI water volume and the atenolol aborption in the presence of $\mathrm{AJ}$ in rats. Water absorption was highest in purified water, followed by saline and isosmotic mannitol solution, and the lowest in AJ, confirming that water absorption is indeed osmolality-dependent. Interestingly, AJ showed apparent water secretion into the intestinal lumen. The intestinal concentration of FD-4, a nonpermeable compound, after administration in AJ was lower than the initial concentration, whereas that in purified water was greater than the initial concentration. Further, the fraction of atenolol absorbed in intestine was significantly lower in AJ or hyperosmotic mannitol solution (adjusted to the osmolality of $\mathrm{AJ}$ ) than after administration in purified water. Comparable results were observed in an in vivo pharmacokinetic study in rats. Our results indicate that orally administered AJ has a capacity to modulate luminal water volume depending on the osmolality, and this effect may result in significant AJ-atenolol interactions.

\section{Introduction}

Drug-drug and food-drug interactions are common causes of adverse drug events. Therefore, understanding drug interactions is important for the clinical use of a drug safely and effectively. In recent years, increasing numbers of interactions between beverages and drugs have been reported. For example, fruit juice (FJ) can alter the intestinal absorption of various drugs, potentially altering the pharmacokinetic behavior of orally administered drugs. Mechanisms underlying beverage-drug interactions involve the inhibition of functional proteins, such as cytochrome P450 (P450)3A and organic anion transporting polypeptide (OATP) 2B1 (Bailey et al., 1991; Shirasaka et al., 2011, 2013). Interestingly, CYP3A-based drug interaction with beverages is confined to grapefruit juice (GFJ), which inhibits CYP3A metabolic activity and thus elevates the bioavailability of drugs that undergo intestinal metabolism mediated by CYP3A (Bailey et al., 1989, 1991; He et al., 1998; Fukuda et al., 2000). On the other hand, OATP2B1mediated drug interaction with beverages has been shown by coadministration with GFJ, orange juice (OJ), apple juice (AJ), and, most recently, green tea, which inhibits OATP2B1 transport activity, resulting

This work was supported in part by a grant-in-aid for Challenging Exploratory Research from the Japan Society for the Promotion of Science (JSPS) [Grant 16K15165] and research grant from the Japan Research Foundation for Clinical Pharmacology [Grant 2017A13].

${ }^{1}$ Y.F. and Y.S. contributed equally to this work.

https://doi.org/10.1124/dmd.118.084483. in a decrease of the bioavailability of substrate drugs (Dresser et al., 2002; Misaka et al., 2014; Yu et al., 2017; Abe et al., 2018).

Naringin and hesperidin, two of the main constituent flavonoids of GFJ and OJ, are believed to be major inhibitors of intestinal drug transport mediated by OATP2B1 (Shirasaka et al., 2009, 2010, 2013); however, the major inhibitor of OATP2B1 in AJ has not been identified yet; several components have been reported to show inhibitory effects, but their additive actions cannot quantitatively explain the direct inhibitory effect of AJ on OATP2B1 (Shirasaka et al., 2013).

It is noteworthy that decreased bioavailability of atenolol and nadolol was clinically observed when these drugs were coadministered with AJ and green tea, respectively, although neither of these drugs is a substrate of OATP2B1 (Jeon et al., 2013; Misaka et al., 2014). Moreover, no significant change in the plasma concentrations of pravastatin and glibenclamide was observed when they were administered together with GFJ, although they are OATP2B1 substrate drugs (Lilja et al., 1999, 2007). These inconsistent findings raise the possibility that some other, unidentified mechanism of beverage-drug interaction may exist.

In a recent study, we showed that the solution osmolality influences gastrointestinal (GI) water volume (Ichijo et al., 2017). Such osmolalitydependent variations in GI water volume may lead to changes in GI drug concentration, resulting in altered absorption characteristics, particularly for low-permeability drugs. Indeed, we found that greater absorption of atenolol in rats was observed with purified water than with isosmotic solution. Thus, we hypothesized that osmolality-dependent changes in water absorption may explain the decrease in the plasma concentration of certain drugs by coadministration with FJ in humans.

ABBREVIATIONS: AJ, apple juice; AUC, area under the plasma concentration-time curve; $C_{\max }$, maximum plasma concentration; FD-4, fluorescein isothiocyanate-dextran 4000; FJ, fruit juice; GFJ, grapefruit juice; GI, gastrointestinal; OATP, organic anion transporting polypeptide; OJ, orange juice; P450, cytochrome 450 . 
In this study, we examined the idea that osmolality-dependent variations in GI water volume resulting from ingested AJ could influence drug absorption characteristics by altering the drug concentration in the GI tract by means of various in vitro, in situ, and in vivo experimental methods by using atenolol, which clinically interacts with AJ. Our results indicate that the luminal water volume is markedly affected by the osmolality of ingested AJ, and this effect contributes substantially to the AJ-atenolol interaction.

\section{Materials and Methods}

Materials. We purchased atenolol from Wako Pure Chemical Industries, Ltd (Osaka, Japan), fluorescein isothiocyanate-dextran 4000 (FD-4) from SigmaAldrich Company (St. Louis, MO), $\left[{ }^{3} \mathrm{H}\right]$ estrone-3-sulfate ammonium salt (E3S, $54 \mathrm{Ci} / \mathrm{mmol}$ ) from PerkinElmer Life Science (Boston, MA); apple juice (AJ) (Tropicana; $100 \%$ pure at normal strength) from a supermarket in Hachioji city, Japan. All other compounds and reagents were obtained from Wako Pure Chemical Industries, Ltd., Nacalai Tesque, Inc. (Kyoto, Japan), or Sigma-Aldrich Company.

Animals. Male Wistar rats were purchased from Tokyo Laboratory Animals Science Co., Ltd. (Tokyo, Japan). All animal experimental protocols were reviewed and approved by the Committee of Animal Care and Welfare of Tokyo University of Pharmacy and Life Sciences.

Measurement of Osmolality of Experimental Solutions. Atenolol or FD-4 was dissolved in purified water, saline $(0.9 \% \mathrm{NaCl})$, AJ, isosmotic mannitol solution (5\% D-mannitol), or hyperosmotic mannitol solution (adjusted to $750 \mathrm{mOsm} / \mathrm{kg}$ corresponding to the osmolality of AJ). For osmolality measurements of experimental solutions, saline, isosmotic solution, hyperosmotic solution, and AJ were centrifuged at $3000 \mathrm{~g}$ for 15 minutes, and the supernatant was collected after filtration. The osmotic pressure of collected supernatant was measured using a cryoscopic osmometer, the OSMOMAT 030-D (Gonotec $\mathrm{GmbH}$, Berlin, Germany).

Uptake Experiments in HEK293/OATP2B1 Cells. HEK293 cells stably expressing human OATP2B1 (HEK293/OATP2B1 cells) and HEK293 cells into which only a vector was introduced (HEK293/mock cells) were used between passage numbers $20-40$ and plated at a density of $10 \times 10^{4}$ cells $/ \mathrm{cm}^{2}$ in 24-well plates coated with poly-L-lysine (Kusuhara et al., 2012). They were cultured for 2 to 3 days to reach $80 \%-90 \%$ confluence. For uptake experiments, culture medium was aspirated, and each well was rinsed with Krebs-Rinber-Henseleit (KRH) buffer (pH 7.4) and preincubated with KRH buffer for 15 minutes at $37^{\circ} \mathrm{C}$. The uptake was initiated by replacing the preincubation buffer with $\mathrm{KRH}$ buffer containing atenolol $(10 \mu \mathrm{M})$ or $\left[{ }^{3} \mathrm{H}\right]$ estrone-3-sulfate $(0.2 \mu \mathrm{Ci} / \mathrm{ml})$ for 30 minutes at $37^{\circ} \mathrm{C}$. The uptake was terminated by washing with ice-cold $\mathrm{KRH}$ buffer; the cells were then solubilized with $1 \mathrm{~N} \mathrm{NaOH}$ and subsequently neutralized with $1 \mathrm{~N} \mathrm{HCl}$. Timedependent uptake was examined to identify the initial rate period (data not shown).

Data analyses for uptake amount (picomoles per milligram protein), uptake rate (picomoles per minute per milligram protein), and uptake clearance (microliters per minute per milligram protein) were carried out in accordance with previous reports (Ichijo et al., 2017).

In Situ Intestinal Closed-Loop Experiment. An in situ intestinal closed-loop study was carried out as described previously (Ichijo et al., 2017). Male Wistar rats (7 to 8 weeks old; fasted overnight) were anesthetized using a mixture of medetomidine, midazolam, and butorphanol. The abdominal cavity was opened, and an intestinal loop (jejunum, $10 \mathrm{~cm}$; ileum, $10 \mathrm{~cm}$; colon, $7 \mathrm{~cm}$ ) was made by cannulation with silicone tubing into both ends of the jejunum, ileum and colon. Test compounds (FD-4 or atenolol) were dissolved in purified water, saline, isosmotic solution, hyperosmotic solution, or AJ. One milliliter of test solution $(10 \mu \mathrm{M})$ was introduced into the intestinal loop, and both ends of the loop were ligated. At the designated times (10,30, or 60 minutes), test solution in the loop was collected by flushing with air (for measuring the luminal concentration of test compound $(\mu \mathrm{M})$ in each intestinal loop, $\left.C_{\text {out }}\right)$ and then made up to $10 \mathrm{ml}$ with buffer solution (for measuring the amount of test compound (micormoles) in each intestinal loop, $X_{\text {out }}$ ). The volume of luminal water (milliliters) in each intestinal segment $\left(V_{\text {water }}\right)$ was calculated using the following equation:

$$
V_{\text {water }}=\frac{X_{\text {out }}}{C_{\text {out }}}
$$

The fraction absorbed of test compound was calculated based on the value of $X_{\text {out }}$, and final results were normalized to the surface area calculated from the length of each intestinal segment and the radii of small and large intestines, which were reported to be 0.178 and $0.252 \mathrm{~cm}$, respectively (Fagerholm et al., 1997).

In Vivo Pharmacokinetic Study in Rats. Male Wistar rats were anesthetized, the right jugular veins were cannulated with silicone tubing $(100-00 \mathrm{~N} ; 0.5 \mathrm{~mm}$ I.D., $1.0 \mathrm{~mm}$ O.D.; Kaneka Medical Products, Osaka, Japan). After fully awaken from the anesthesia, the rats were orally administered with test compound solution of atenolol ( $1 \mathrm{mg} / \mathrm{kg}, 0.25 \mathrm{mg} / \mathrm{ml})$ by gavage. The rats were freely moving during the experiment. Blood samples $(500 \mu \mathrm{l})$ were collected from the cannula into heparinized tubes at designated times (up to 540 minutes) and centrifuged at $3000 \mathrm{rpm}$ for 10 minutes. The resultant plasma was stored at $-30^{\circ} \mathrm{C}$ until analysis.

Plasma concentration-time curves of atenolol were plotted and analyzed. The area under the plasma concentration-time curve from 0 to 9 hour $\left(\mathrm{AUC}_{0-9}\right)$ and from 0 to infinite $\left(\mathrm{AUC}_{0-\infty}\right)$ was calculated by the trapezoidal method using noncompartmental analysis with the MOMENT program (Yamaoka et al., 1978). The maximum plasma concentration $\left(C_{\max }\right)$ and the time to reach $C_{\max }\left(t_{\max }\right)$ were obtained directly from the experimental data. The elimination half-life $\left(t_{1 / 2}\right)$ was calculated based on the terminal elimination rate constant determined by loglinear regression of the final data points (at least 3 ).

Analytical Methods. Concentrations of FD-4 were measured using a microplate fluorescence reader (VarioskanTM Flash 2.4; Thermo Fisher Scientific Inc., Kanagawa, Japan) at excitation/emission wavelengths of 492/515 nm. Radioactivity was determined using a liquid scintillation counter (LSC) (Tri-Carb 3110TR; PerkinElmer, Waltham, MA).

The concentration of atenolol was measured using a LC-MS/MS system consisting of a AB-Sciex API 3200 triple-quadrupole mass spectrometer (AB SCIEX, Foster City, CA) coupled with a LC-20AD ultra-fast liquid chromatography (UFLC) system (Shimadzu Company, Kyoto, Japan). An Agilent ZORBAX Eclipse Plus $\left(\mathrm{C}_{18}, 50 \times 2.1 \mathrm{~mm}, 5 \mu \mathrm{m}\right)$ was used as the analytical column. A mobile phase composed of $0.1 \%$ formic acid (A) and acetonitrile (B) was used at a flow rate of $0.4 \mathrm{ml} / \mathrm{min}$ with a gradient elution starting from $2 \% \mathrm{~B}$ for $0-1.5$ minutes, $2.0 \%-80 \%$ B for $1.5-2.0$ minutes, $80 \%$ B for $2.0-4.5$ minutes, $80 \%-50 \%$ B for 4.5-5.0 minutes, $50 \%$ B for $5.0-6.0$ minutes, $50 \%-2.0 \%$ B for $6.0-6.5$ minutes, and $2 \%$ B for 6.5-7.0 minutes. The mass transitions (Q1/Q3) of $\mathrm{m} / \mathrm{z}, 267.2 / 145.2$ and 260.1/116.2 were used for atenolol and propranolol (as an internal standard), respectively. Analyst software version 1.4.2 (AB SCIEX) was used for data analysis.

Statistical Analysis. Data are given as the mean of values obtained in at least three experiments with the standard error (S.E.M.). A basic statistical analysis was performed using Microsoft Office Excel 2016 (Microsoft Corporation, Redmond, WA). The statistical significance of differences for two-group and multiple comparisons was evaluated using the unpaired Student's $t$ test and one-way ANOVA, followed by Dunnett's or Tukey's tests, respectively. A probability of less than $0.05(P<0.05)$ was considered statistically significant.

\section{Results}

Uptake of Atenolol and Estrone 3-Sulfate by HEK293/OATP2B1. To examine whether atenolol is a substrate of OATP2B1, we measured the uptake of atenolol and estrone 3-sulfate (E3S) by HEK293/OATP2B1 cells (Fig. 1). In positive control studies, the uptake of typical substrates E3S by HEK293/OATP2B1 cells was significantly greater than that by HEK293/mock cells. In contrast, the uptake of atenolol $(10 \mu \mathrm{M})$ was comparable in HEK293/OATP2B1 and HEK293/mock cells. These findings indicate that atenolol is not a substrate of OATP2B1.

Impact of Solution Osmolality on Luminal Water Volume in Rats. To study the impact of solution osmolality on GI water movement, the time course of the water volume in jejunum was examined after the ingestion of purified water, saline, isosmotic solution, and AJ (Fig. 2). Water absorption was greatest in purified water, followed by saline, whereas no water absorption was apparently observed with isosmotic solution. In the case of AJ, which is hyperosmolar, secretion of water into the lumen was observed. When the osmolality of each solution was measured, AJ (749.6 \pm $19.8 \mathrm{mOsm} / \mathrm{kg}$ ) showed high osmolality, whereas saline $(286.6 \pm 0.4$ $\mathrm{mOsm} / \mathrm{kg}$ ) and isosmotic solution (303.9 $\pm 11.2 \mathrm{mOsm} / \mathrm{kg}$ ) exhibited isotonicity. These results clearly indicate that intestinal absorption of water is 


\section{A Atenolol}

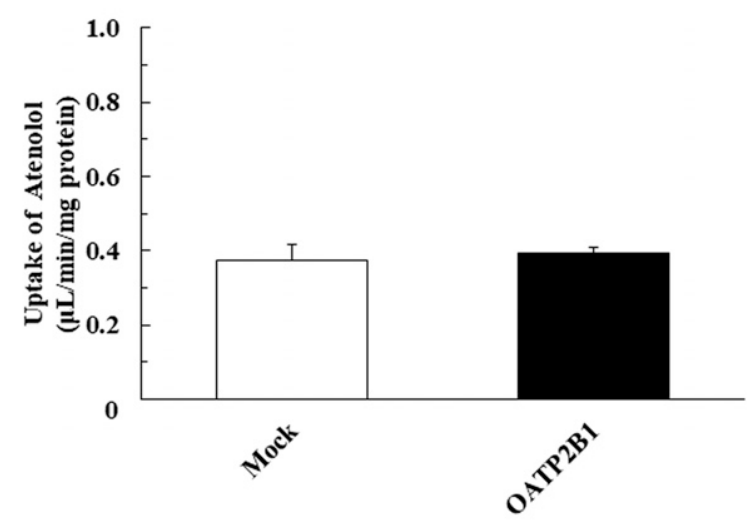

\section{B Estrone-3-Sulfate}

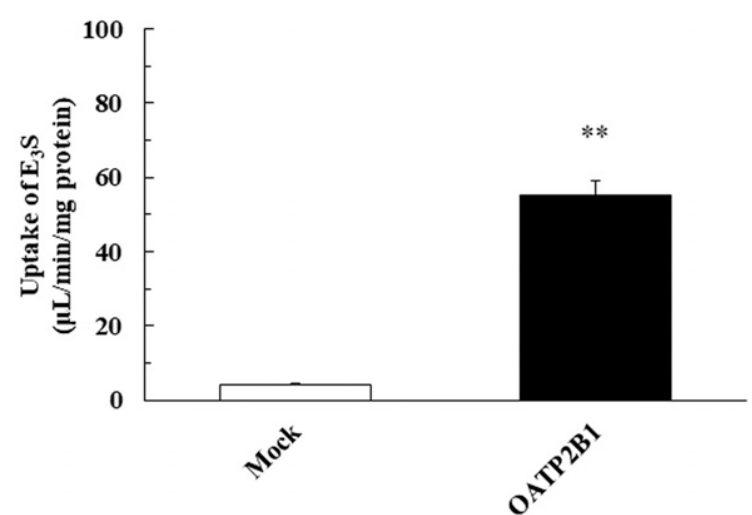

Fig. 1. Uptake of atenolol and estrone 3-sulfate by HEK293/OATP2B1 cells. The uptakes of (A) atenolol and (B) $\left[{ }^{3} \mathrm{H}\right]$ estrone-3-sulfate by HEK293/OATP2B1 (closed bars) and HEK293/mock (open bars) cells were measured for 30 minute at $37^{\circ} \mathrm{C}$ and $\mathrm{pH}$ 7.4. The applied concentrations of atenolol and $\left[{ }^{3} \mathrm{H}\right]$ estrone-3-sulfate were $10 \mu \mathrm{M}$ and $0.2 \mu \mathrm{Ci} / \mathrm{ml}$, respectively. $* P<0.05 ; * * P<0.01$, significantly different from HEK293/mock cells. Data are shown as the mean \pm S.E.M. $(n=3)$.

osmolality-dependent. In other words, the solution osmolality is involved in the water volume in the intestine.

Impact of Solution Osmolality on Intestinal Concentration of FD-4 in Rats. To investigate the impact of the solution osmolality on the intestinal drug concentration, the concentration of FD-4 was measured at 30 minutes in jejunum after ingestion of purified water, saline, isosmotic solution, and AJ (Fig. 3). As shown in Fig. 3, intestinal concentrations of FD-4 after ingestion of these solutions were $20.3 \pm$ $1.7,12.8 \pm 1.2,9.81 \pm 0.87$, and $7.01 \pm 0.57 \mu \mathrm{M}$, respectively. Interestingly, the intestinal concentration of FD-4 after administration in purified water was markedly greater than the initial concentration $(10 \mu \mathrm{M})$, whereas the concentration of FD-4 after administration in AJ was lower than the initial concentration. No significant difference was shown between saline and isosmotic solution.

Impact of Solution Osmolality on Intestinal Absorption of Atenolol in Rats. To study the effect of water movement on intestinal absorption of atenolol, the luminal concentration and fraction of atenolol absorbed in jejunum, ileum, and colon were determined after administration in purified water, $\mathrm{AJ}$, and hyperosmotic solution (adjusted to the same osmolality as AJ) (Fig. 4). In Fig. 4A, the water volume 30 minutes after administration of atenolol in purified water was $60.9 \% \pm 4.5 \%$ (jejunum), $74.5 \% \pm 9.2 \%$ (ileum), and $25.5 \% \pm 4.5 \%$ (colon), whereas the corresponding values after administration in $\mathrm{AJ}$ were $154.7 \% \pm$ $11.4 \%$ (jejunum), $139.1 \% \pm 4.3 \%$ (ileum), and $141.6 \% \pm 12.8 \%$ (colon), respectively. Thus, the water volume was remarkably reduced

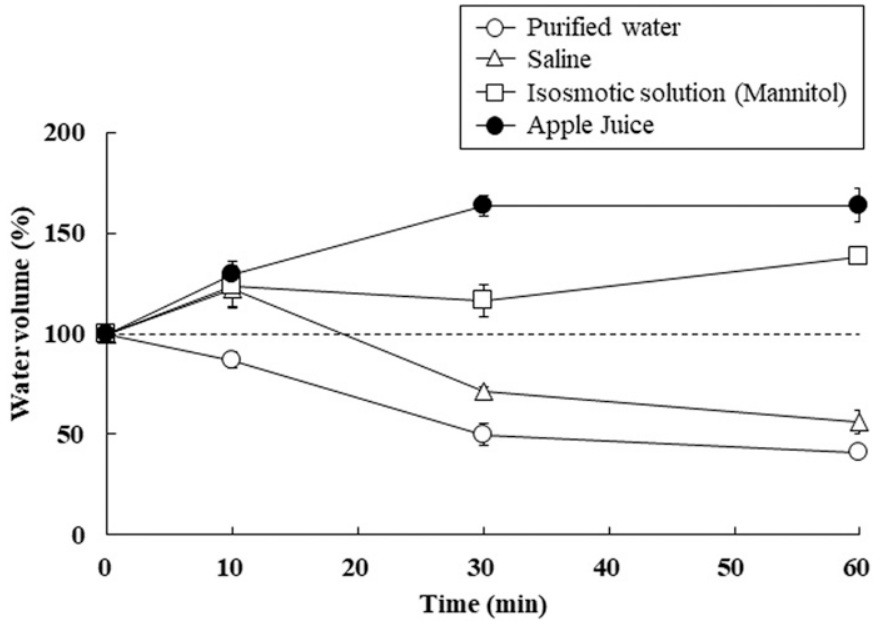

Fig. 2. Influence of solution osmolality on the time course of water volume in rat jejunum. The water volume in jejunum was determined by means of the in situ closed loop method using FD-4 $(10 \mu \mathrm{M})$ in various solvents (purified water, saline, isosmotic mannitol solution and apple juice) at 10,30 and 60 minute at $37^{\circ} \mathrm{C}$. Data are means \pm S.E.M. $(n=6-9)$.

after administration in purified water, but it conversely increased after administration in AJ, in agreement with the results shown in Fig. 2. As shown in Fig. 4B, the luminal concentrations of atenolol 30 minutes after administration in purified water were $11.1 \pm 0.9$ (jejunum), $10.3 \pm 1.5$ (ileum), and $21.5 \pm 3.0 \mu \mathrm{M}$ (colon), whereas those after administration in AJ were $6.43 \pm 0.6$ (jejunum), $6.72 \pm 0.4$ (ileum), and $5.6 \pm 0.3 \mu \mathrm{M}$ (colon). On the other hand, the values of the fraction absorbed of atenolol 30 minutes after administration in purified water were $34.4 \% \pm 3.9 \%$ (jejunum), $26.5 \% \pm 2.3 \%$ (ileum), and $38.4 \% \pm 15.8 \%$ (colon), whereas those after administration in $\mathrm{AJ}$ were $12.3 \% \pm 1.9 \%$ (jejunum), $13.3 \% \pm 2.6 \%$ (ileum), and $8.26 \% \pm 0.4 \%$ (colon) (Fig. 4C). Thus, the intestinal absorption of atenolol in every intestinal segment was less after administration in $\mathrm{AJ}$ than after administration in purified water.

To consider the impact of solution osmolality on intestinal absorption of atenolol, we next carried out a comparison study on the luminal

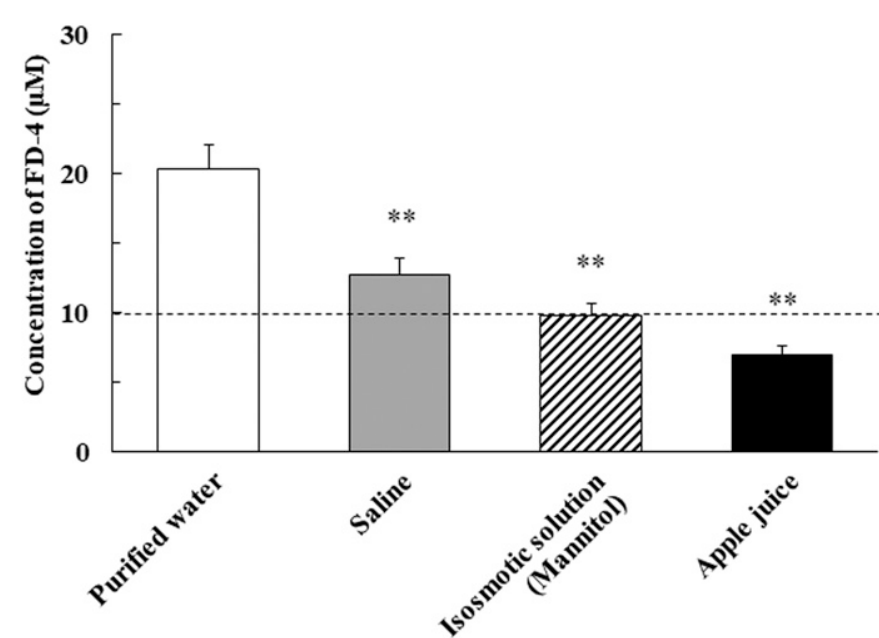

Fig. 3. Influence of solution osmolality on intestinal concentration of FD-4 in rats. The jejunum concentrations of FD-4 after administration in various solvents (purified water, saline, isosmotic solution and apple juice) was measured by means of an in situ closed loop method for 30 minute at $37^{\circ} \mathrm{C}$. The statistical significance of differences between the different conditions was evaluated using one-way ANOVA followed by Dunnett's test. $* P<0.05$; $* * P<0.01$, significantly different from purified water. Data are shown as means \pm S.E.M. $(n=6-9)$. 

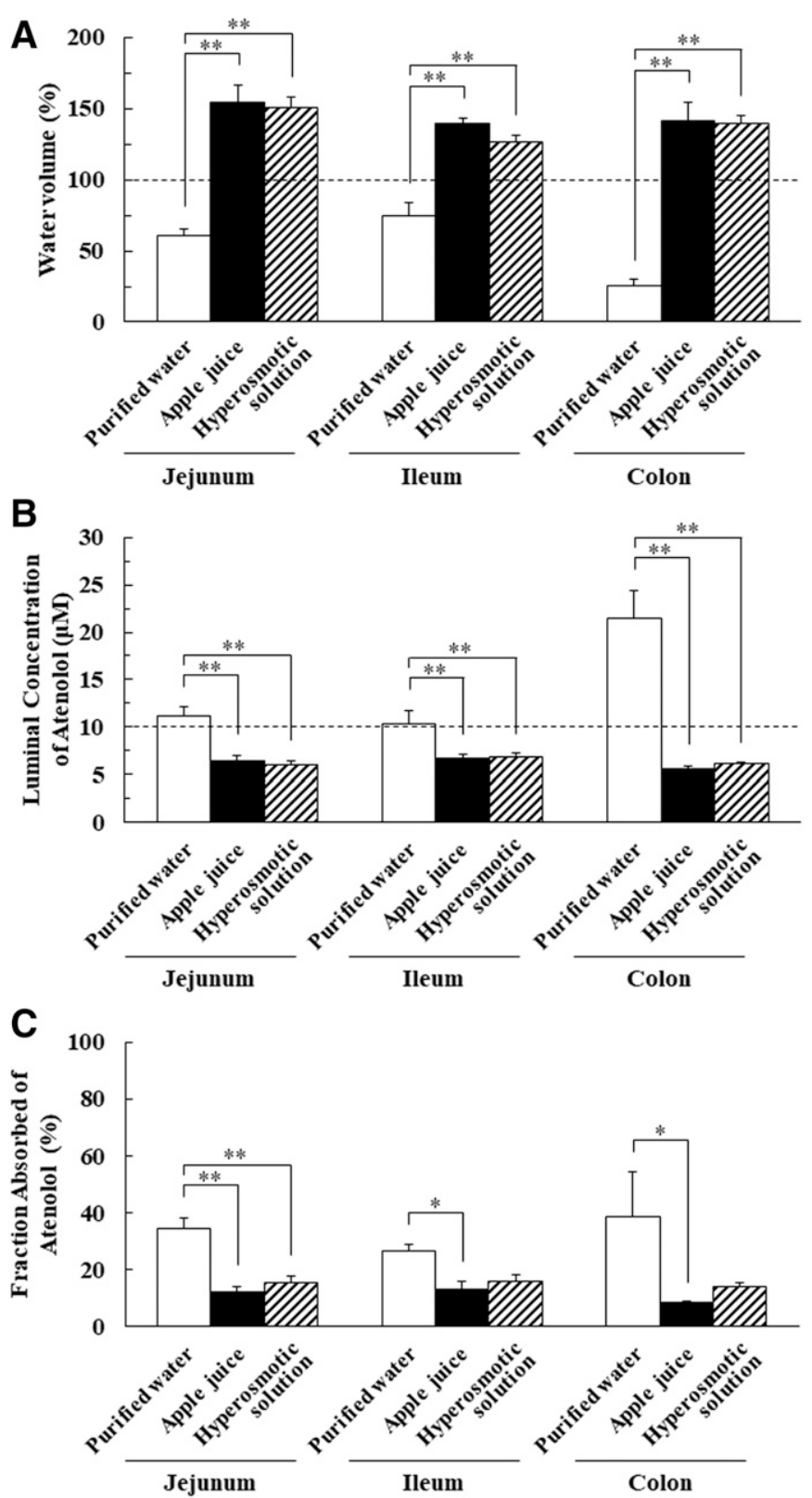

Fig. 4. Impact of solution osmolality on intestinal concentration and fraction absorbed of atenolol in rats. (A) Water volume, (B) luminal concentration of atenolol and $(C)$ fraction absorbed of atenolol in jejunum, ileum and colon after administration of atenolol $(10 \mu \mathrm{M})$ in purified water, apple juice (AJ) or hyperosmotic mannitol solution were determined by means of the in situ closed loop method for 30 minute at $37^{\circ} \mathrm{C}$. The statistical significance of differences between the different conditions was evaluated using one-way ANOVA followed by Tukey's multiple comparison test. Significantly different at $* P<0.05 ; * * P<0.01$. Data are shown as means \pm S.E.M. $(n=6-9)$.

concentration and the fraction absorbed of atenolol in jejunum, ileum, and colon after ingestion of AJ and hyperosmotic solution (Fig. 4). As shown in Fig. 4, after the ingestion of atenolol in hyperosmotic solution, the water volume was $151.0 \% \pm 6.8 \%$ (jejunum), $126.5 \% \pm 4.5 \%$ (ileum), and $139.8 \% \pm 5.0 \%$ (colon); the luminal concentration of atenolol was $6.1 \pm 0.3$ (jejunum), $6.9 \pm 0.4$ (ileum), and $6.1 \pm 0.1 \mu \mathrm{M}$ (colon); and the values of fraction absorbed of atenolol were $15.5 \% \pm$ $2.1 \%$ (jejunum), $15.6 \% \pm 2.4 \%$ (ileum), and $13.9 \% \pm 1.6 \%$ (colon). Thus, no significant difference in the fraction absorbed of atenolol was observed between $\mathrm{AJ}$ and hyperosmotic solution.
Effect of Solution Osmolality on Oral Absorption of Atenolol in Rats. When atenolol $(1 \mathrm{mg} / \mathrm{kg})$ was orally administered with $1 \mathrm{ml}$ of purified water to rats, the $\mathrm{AUC}_{0-9}$ and $C_{\max }$ of atenolol were $278 \pm$ $34 \mathrm{ng} \cdot \mathrm{h} / \mathrm{ml}$ and $72.3 \pm 7.2 \mathrm{ng} / \mathrm{ml}$, respectively (Fig. 5; Table 1). Simultaneous administration of AJ with atenolol significantly decreased the $\mathrm{AUC}_{0-9}$ and $C_{\max }$ to $63.2 \%$ and $59.9 \%$, respectively (Fig. 5; Table 1). Similarly, simultaneous administration of hyperosmotic solution significantly decreased $\mathrm{AUC}_{0-9}$ and $C_{\max }$ of atenolol to $26.4 \%$ and $24.5 \%$, respectively; however, AJ and hyperosmotic solution did not significantly alter $t_{1 / 2}$ of atenolol (Table 1 ).

\section{Discussion}

Recently, it was reported that AJ ingestion markedly decreased systemic availability of atenolol (Jeon et al., 2013); however, because genetic variations in OATP2B1 were not associated with the variability to the pharmacokinetics of atenolol, inhibition of OATP2B1 cannot account for this clinical interaction. This consideration is strongly supported by our in vitro study that atenolol was found not to be a substrate of OATP2B1 (Fig. 1). Therefore, it is possible that an unidentified mechanism, other than OATP2B1 inhibition, of AJ-atenolol interaction may exist. On the other hand, we more recently showed that the solution osmolality influences the GI water volume, and we suggested that this might indirectly affect intestinal drug absorption (Ichijo et al., 2017). In this study, we investigated whether osmolality-dependent water movement can account for the reduction in the systemic exposure to atenolol by coadministration of $\mathrm{AJ}$ in humans.

As shown in Fig. 2, the water movement after administration in saline was not altered compared with that in purified water, whereas a distinct difference was observed between purified water and isosmotic solution. Because intestinal epithelium contains various types of $\mathrm{NaCl}$ transport systems (e.g., $\mathrm{Na}^{+} / \mathrm{H}^{+}$and $\mathrm{Cl}^{-} / \mathrm{HCO}_{3}^{-}$exchangers), it is possible to consider that water absorption was stimulated by decreasing the luminal osmolality of saline resulted from $\mathrm{Na}^{+}$and/or $\mathrm{Cl}^{-}$absorption; however, the water absorption behavior for 10 minutes seems slightly different between saline and purified water. This apparent discrepancy may be explained by initial osmolality, that is, isotonic condition, in saline. This explanation is bolstered by the fact that the water absorption 10 minutes after ingestion of saline and isosmotic solution exhibits the same behavior (Fig. 2). In contrast, the secretion of water into the lumen

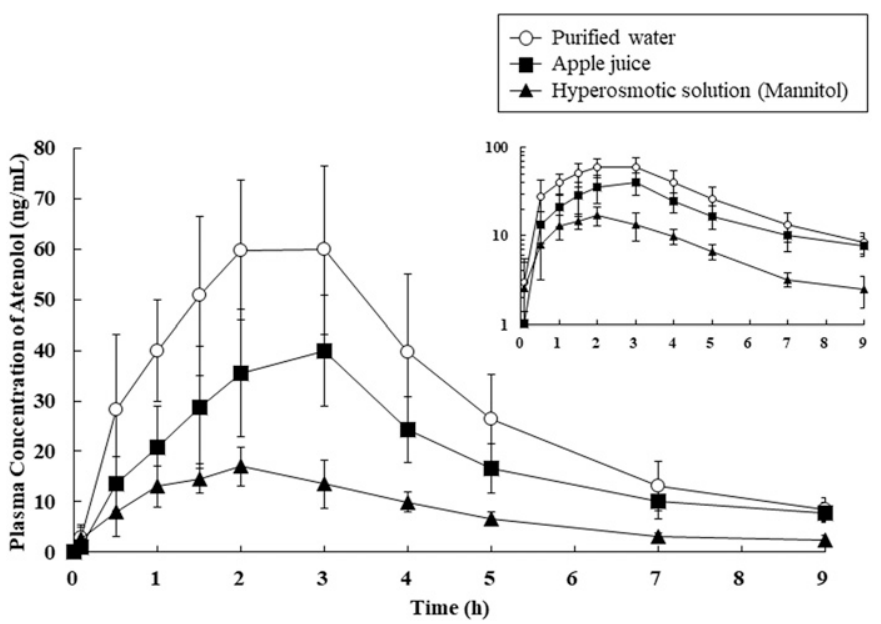

Fig. 5. Mean plasma concentration-time profiles of atenolol in rats after oral administration. Atenolol $(1 \mathrm{mg} / \mathrm{kg}, 0.25 \mathrm{mg} / \mathrm{ml})$ was orally administered with purified water (open circles), apple juice (closed squares) or hyperosmotic mannitol solution (closed triangles). Data are shown as the mean \pm S.D. $(n=6)$. 
TABLE 1

Pharmacokinetics of atenolol after oral administration to rats

Atenolol $(1 \mathrm{mg} / \mathrm{kg}, 0.25 \mathrm{mg} / \mathrm{ml})$ was orally administered with purified water, apple juice or hyperosmotic mannitol solution. The statistical significance of differences between the different conditions was evaluated using one-way ANOVA followed by Tukey's multiple comparison test.

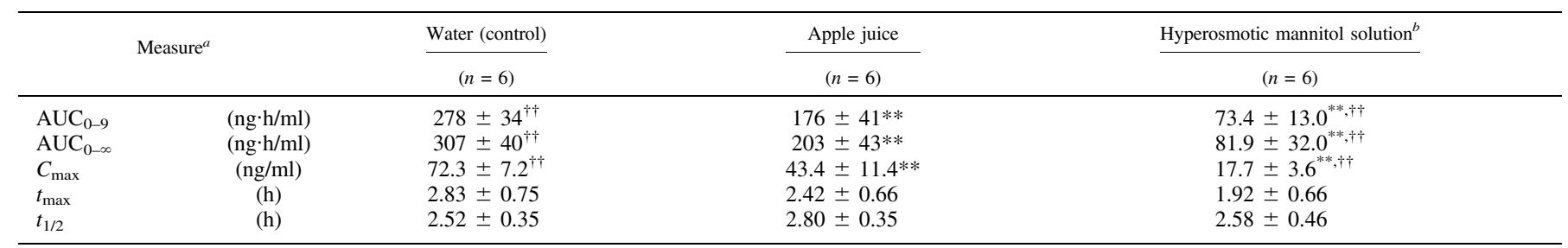

${ }^{a} \mathrm{AUC}$, area under plasma concentration-time curve; $C_{\max }$, maximum plasma concentration; $t_{\max }$, time to reach maximum plasma concentration; $t_{1 / 2}$, elimination half-life.

${ }^{b}$ Hyperosmotic mannitol solution was adjusted to the same osmolality as AJ.

$* P<0.05 ; * * P<0.01$, significantly different from control values.

$\dagger P<0.05 ; \dagger \uparrow P<0.01$, significantly different from values with AJ. Data are shown as means \pm S.D. $(n=6)$

was observed with AJ. The osmotic concentration of AJ is due mainly to short-chain carbohydrates, such as glucose, fructose, and sucrose. In particular, fructose is reportedly contained at the highest concentration in AJ, among the constituent carbohydrates (Murray et al., 2014). Because fructose is known to be a poorly permeable substance, even though it is a substrate of sugar transporters GLUT2 and GLUT5, the luminal osmolality of AJ may be maintained at a high level (Ferraris et al., 2018). This simply means that when AJ is orally ingested to rats, secretion of water into the lumen may be induced due to the highosmolality environment in the GI tract. These considerations are supported by magnetic resonance imaging (MRI) analyses, which revealed that a solution of fructose, but not glucose, led to a remarkable increase of luminal fluid volume (Murray et al., 2014; Grimm et al., 2018). Osmolality-dependent increases in water secretion may reduce luminal drug concentration, possibly resulting in decreased drug absorption. Indeed, after administration in AJ, the intestinal concentration of FD-4, which is non-permeable, was lower than the initial dosing concentration, while that after administration in purified water exceeded the initial concentration (Fig. 3). Very interestingly, the intestinal absorption of atenolol was significantly lower after administration in AJ than after administration in purified water (Fig. 4C). This can be explained by the opposite behaviors of GI water dynamics after ingestion of purified water and AJ. In the former case, rapid water absorption may result in an increase of drug concentration, leading to increased absorption because of the elevated concentration gradient. In the latter case, greater water secretion may result in a decrease of drug concentration, leading to decreased absorption due to the lower concentration gradient.

To verify these findings, we carried out an in vivo pharmacokinetic study of atenolol in rats. The plasma concentration of atenolol following oral administration was significantly reduced by coadministration of AJ (Fig. 5; Table 1). This is in good agreement with a clinical report describing a decrease of AUC and $\mathrm{C}_{\max }$ of atenolol upon coadministration with AJ in humans (Jeon et al., 2013). These findings raise the possibility that osmolality-dependent water movement is the major determinant of AJ-atenolol interaction in humans as well as rats. However, we don't have any direct evidence from this study to show the quantitative effect of osmolality on GI water movement. The direct relationship between osmolality and water movement should be examined and is an issue for further investigation.

Recent investigations have shown that intestinal absorption of drugs can be reduced through the inhibition of OATP2B1 by FJ and its components (Dresser et al., 2002; Shirasaka et al., 2011, 2013). AJ was also reported to inhibit OATP2B1, although the major inhibitor of OATP2B1 in AJ has not been identified yet (Jeon et al., 2013; Shirasaka et al., 2013). However, our results shown in Fig. 1 indicated that inhibition of OATP2B1 cannot account for the AJ-atenolol interaction, because atenolol was found not to be a substrate of OATP2B1 (Fig. 1). On the other hand, atenolol is reported to be a substrate of OCT1/SLC22A1 and PMAT/SLC29A4, both of which are expressed in the intestine (Mimura et al., 2015, 2017). Because OCT1 and PMAT are reportedly inhibited by phloretin and quercetin, which are major constituent flavonoids of AJ, these molecules are candidates for the key determinant of such interactions. However, the concentrations of phloretin $(0.233 \mu \mathrm{M})$ and quercetin $(0.464 \mu \mathrm{M})$ in AJ are below the $I C_{50}$ values for OCT1 (38.0 and $48.0 \mu \mathrm{M}$, respectively) and PMAT (33.3 and 116.3 $\mu \mathrm{M}$, respectively) (Shirasaka et al., 2013; Mimura et al., $2015,2017)$. Therefore, it is unlikely that these flavonoids are major contributors to OCT1- and PMAT-mediated atenolol interactions involving AJ.

To clarify the primary mechanisms and their contributions to the AJ-atenolol interaction, further studies on the intestinal concentration and absorption of atenolol after administration in $\mathrm{AJ}$ and hyperosmotic solution (adjusted to osmolality of AJ) were carried out (Figs. 4 and 5). As a result, the fraction absorbed of atenolol was comparable between AJ and hyperosmotic solution (Fig. 4). These results indicate that factors other than osmolality (e.g., OATP and other transporters) do not make any major contribution to the AJ-atenolol interaction. In other words, osmolality-dependent water movement may be the major determinant of AJ-atenolol interaction. Meanwhile, in Fig. 5 and Table 1, similar pattern but statistically-significant difference in in vivo pharmacokinetics of atenolol was observed between AJ and hyperosmotic solution. This may be explained by the attenuation of the influence of water secretion on atenolol absorption due to a decrease in the osmolality of ingested AJ. Because AJ contains various ions such as sodium and potassium, the osmotic concentration of AJ may be due also to these ions as well as carbohydrates. In this case, similar to active transport of $\mathrm{NaCl}$ in saline, ion transport systems expressed in intestinal epithelium must lead to a lowering in the osmolality of ingested AJ as a result of ion absorption. Further studies are required to examine the potential contribution of these transport systems to osmolality-dependent water movement and drug absorption in intestine.

We have little information about the effect of solution osmolality on intestinal drug absorption in human in vivo. Therefore, to consider the contribution of osmolality to AJ-atenolol interaction in human, it would be important to examine the species differences in the impact of solution osmolality on luminal water volume and drug absorption between human and rat. However, in a recent clinical study, the plasma concentrations of five drugs (sulfasalazine, rosuvastatin, glibenclamide, celiprolol and sumatriptan) were reportedly decreased upon coadministration of GFJ, 
though no significant changes in the plasma concentrations of these drugs were observed upon coadministration of atorvastatin, a strong inhibitor of OATP2B1 (Kashihara et al., 2017). This implies that osmolalitydependent water movement may account for the decrease in plasma concentration of various drugs by coadministration of not only AJ, but also GFJ and other beverages.

In conclusion, our findings indicate that osmolality-dependent variations in GI water volume may indirectly affect the absorption characteristics of atenolol by altering its concentration in the GI tract. Although contributions of influx (e.g., OATP, PMAT) and/or efflux (e.g., P-glycoprotein) transporter molecules cannot be ruled out, this study strongly supports our idea that the luminal water volume is influenced by the solution osmolality, and this effect may play a role in various beverage-drug interactions.

Ethical Statement. The authors declare that they have no conflict of interest. This work did not involve studies with human subjects.

\section{Acknowledgments}

We would like to thank Dr. Hiroyuki Kusuhara and Dr. Kazuya Maeda of University of Tokyo for kindly providing HEK293/OATP2B1 cells.

\section{Authorship Contributions}

Participated in research design: Shirasaka, Inoue.

Conducted experiments: Funai, Shirasaka, Ishihara, Ichijo, Takemura.

Performed date analysis: Funai, Shirasaka, Ishihara, Ichijo, Takemura, Kishimoto.

Wrote or contributed to the writing of the manuscript: Funai, Shirasaka, Kishimoto, Inoue.

\section{References}

Abe O, Ono T, Sato H, Müller F, Ogata H, Miura I, Shikama Y, Yabe H, Onoue S, Fromm MF, et al. (2018) Role of (-)-epigallocatechin gallate in the pharmacokinetic interaction between nadolol and green tea in healthy volunteers. Eur J Clin Pharmacol 74:775-783.

Bailey DG, Spence JD, Edgar B, Bayliff CD, and Arnold JM (1989) Ethanol enhances the hemodynamic effects of felodipine. Clin Invest Med 12:357-362.

Bailey DG, Spence JD, Munoz C, and Arnold JM (1991) Interaction of citrus juices with felodipine and nifedipine. Lancet 337:268-269.

Dresser GK, Bailey DG, Leake BF, Schwarz UI, Dawson PA, Freeman DJ, and Kim RB (2002) Fruit juices inhibit organic anion transporting polypeptide-mediated drug uptake to decrease the oral availability of fexofenadine. Clin Pharmacol Ther 71:11-20.

Fagerholm U, Lindahl A, and Lennernäs H (1997) Regional intestinal permeability in rats of compounds with different physicochemical properties and transport mechanisms. J Pharm Pharmacol 49:687-690.

Ferraris RP, Choe JY, and Patel CR (2018) Intestinal absorption of fructose. Annu Rev Nutr 38: $41-67$.

Fukuda K, Guo L, Ohashi N, Yoshikawa M, and Yamazoe Y (2000) Amounts and variation in grapefruit juice of the main components causing grapefruit-drug interaction. $J$ Chromatogr $B$ Biomed Sci Appl 741:195-203.
Grimm M, Koziolek M, Saleh M, Schneider F, Garbacz G, Kühn JP, and Weitschies W (2018) Gastric emptying and small bowel water content after administration of grapefruit juice compared to water and isocaloric solutions of glucose and fructose: a four-way crossover MRI pilot study in healthy subjects. Mol Pharm 15:548-559.

He K, Iyer KR, Hayes RN, Sinz MW, Woolf TF, and Hollenberg PF (1998) Inactivation of cytochrome P450 3A4 by bergamottin, a component of grapefruit juice. Chem Res Toxicol 11: $252-259$.

Ichijo K, Oda R, Ishihara M, Okada R, Moteki Y, Funai Y, Horiuchi T, Kishimoto H, Shirasaka Y, and Inoue K (2017) Osmolality of orally administered solutions influences luminal water volume and drug absorption in intestine. J Pharm Sci 106:2889-2894.

Jeon H, Jang IJ, Lee S, Ohashi K, Kotegawa T, Ieiri I, Cho JY, Yoon SH, Shin SG, Yu KS, et al. (2013) Apple juice greatly reduces systemic exposure to atenolol. Br J Clin Pharmacol 75: 172-179.

Kashihara Y, Ieiri I, Yoshikado T, Maeda K, Fukae M, Kimura M, Hirota T, Matsuki S, Irie S, Izumi $\mathrm{N}$, et al. (2017) Small-dosing clinical study: pharmacokinetic, pharmacogenomic (SLCO2B1 and ABCG2), and interaction (atorvastatin and grapefruit juice) profiles of 5 probes for OATP2B1 and BCRP. J Pharm Sci 106:2688-2694.

Kusuhara H, Furuie H, Inano A, Sunagawa A, Yamada S, Wu C, Fukizawa S, Morimoto N, Ieiri I, Morishita M, et al. (2012) Pharmacokinetic interaction study of sulphasalazine in healthy subjects and the impact of curcumin as an in vivo inhibitor of BCRP. Br J Pharmacol 166:1793-1803.

Lilja JJ, Kivistö KT, and Neuvonen PJ (1999) Grapefruit juice increases serum concentrations of atorvastatin and has no effect on pravastatin. Clin Pharmacol Ther 66:118-127.

Lilja JJ, Niemi M, Fredrikson H, and Neuvonen PJ (2007) Effects of clarithromycin and grapefruit juice on the pharmacokinetics of glibenclamide. Br J Clin Pharmacol 63:732-740.

Mimura Y, Yasujima T, Ohta K, Inoue K, and Yuasa H (2015) Functional identification of organic cation transporter 1 as an atenolol transporter sensitive to flavonoids. Biochem Biophys Rep 2:166-171.

Mimura Y, Yasujima T, Ohta K, Inoue K, and Yuasa H (2017) Functional identification of plasma membrane monoamine transporter (PMAT/SLC29A4) as an atenolol transporter sensitive to flavonoids contained in apple juice. J Pharm Sci 106:2592-2598.

Misaka S, Yatabe J, Müller F, Takano K, Kawabe K, Glaeser H, Yatabe MS, Onoue S, Werba JP, Watanabe H, et al. (2014) Green tea ingestion greatly reduces plasma concentrations of nadolol in healthy subjects. Clin Pharmacol Ther 95:432-438.

Murray K, Wilkinson-Smith V, Hoad C, Costigan C, Cox E, Lam C, Marciani L, Gowland P, and Spiller RC (2014) Differential effects of FODMAPs (fermentable oligo-, di-, monosaccharides and polyols) on small and large intestinal contents in healthy subjects shown by MRI. Am J Gastroenterol 109:110-119.

Shirasaka Y, Kuraoka E, Spahn-Langguth H, Nakanishi T, Langguth P, and Tamai I (2010) Species difference in the effect of grapefruit juice on intestinal absorption of talinolol between human and rat. J Pharmacol Exp Ther 332:181-189.

Shirasaka Y, Li Y, Shibue Y, Kuraoka E, Spahn-Langguth H, Kato Y, Langguth P, and Tamai I (2009) Concentration-dependent effect of naringin on intestinal absorption of beta(1)-adrenoceptor antagonist talinolol mediated by p-glycoprotein and organic anion transporting polypeptide (Oatp). Pharm Res 26:560-567.

Shirasaka Y, Shichiri M, Mori T, Nakanishi T, and Tamai I (2013) Major active components in grapefruit, orange, and apple juices responsible for OATP2B1-mediated drug interactions. J Pharm Sci 102:280-288.

Shirasaka Y, Suzuki K, Nakanishi T, and Tamai I (2011) Differential effect of grapefruit juice on intestinal absorption of statins due to inhibition of organic anion transporting polypeptide and/or P-glycoprotein. J Pharm Sci 100:3843-3853.

Yamaoka K, Nakagawa T, and Uno T (1978) Statistical moments in pharmacokinetics. $J$ Pharmacokinet Biopharm 6:547-558.

Yu J, Zhou Z, Tay-Sontheimer J, Levy RH, and Ragueneau-Majlessi I (2017) Intestinal drug interactions mediated by OATPs: a systematic review of preclinical and clinical findings. J Pharm Sci 106:2312-2325.

Address correspondence to: Dr. Yoshiyuki Shirasaka, School of Pharmacy, Tokyo University of Pharmacy and Life Sciences, 1432-1 Horinouchi, Hachioji, Tokyo 192-0392, Japan. E-mail: shira@toyaku.ac.jp 